\section{Judicialização da política pública de assistência farmacêutica e eqüidade}

\author{
"Judicialization" of public health policy for \\ distribution of medicines
}

\begin{abstract}
The supply of medicines in response to court orders or injunctions has become a common practice in the State of São Paulo, Brazil. This "judicialization" of the health system clashes with basic principles of the Brazilian Unified National Health System (SUS), such as equal opportunity to access health services. The aim of this paper is to analyze the legal action used to obtain medicines through the São Paulo State Health Department, from two main angles: judicialization of public policies and breach of the equity principle. This is a descriptive study of legal action taken to obtain medicines through the São State Health Department, as listed in the Electronic Court Docket System for the year 2006. Most cases were filed through private attorneys; $47 \%$ of the patients had obtained their prescriptions through private care; and $73 \%$ of the cases involved patients from the three wealthiest areas in the city of São Paulo. The data demonstrate that such legal action violates key principles of the SUS such as equity, thereby privileging individuals with higher purchasing power and more access to information.
\end{abstract}

Judicial Decisions; Pharmaceutical Care; Drugs; Right to Health
Ana Luiza Chieffi 1

Rita Barradas Barata 2

\section{Introdução}

O fornecimento de medicamentos por ação judicial tornou-se uma prática rotineira nos últimos anos. No Estado de São Paulo, Brasil, os números dessas demandas vêm aumentando consideravelmente. No ano 2006, a Secretaria de Estado da Saúde de São Paulo (SES-SP) gastou, com o cumprimento das decisões judiciais da comarca da capital, 65 milhões de Reais, para atender cerca de 3.600 pessoas. Em comparação, no mesmo ano, ela investiu 838 milhões de Reais no Programa de Medicamentos de Dispensação Excepcional (alto custo), atendendo 380 mil pessoas. Foram gastos aproximadamente 18 mil Reais por paciente com ações judiciais naquele ano, enquanto o Programa de Medicamentos de Dispensação Excepcional consumiu 2,2 mil Reais por paciente 1 .

Após a aprovação da Constituição Federal Brasileira de 1988 tornou-se cada vez mais freqüente a interferência do poder judiciário em questões que, primariamente, são da competência dos poderes executivos ou legislativos. A este novo papel exercido pelo Judiciário na garantia de direitos individuais tem sido atribuída a noção de judicialização.

No campo específico da política de saúde, a judicialização tem se traduzido como a garantia de acesso a bens e serviços por intermédio do recurso a ações judiciais. Com o aumento exponencial das ações e a impossibilidade de previsão orçamentária dos gastos por elas acarretados, os 
gestores do sistema de saúde, nos âmbitos municipal, estadual e federal, tentam resolver de diversas maneiras os impasses criados.

Um dos aspectos da assistência médica que mais tem sido alvo das ações judiciais é a assistência farmacêutica, ou seja, a garantia do acesso a medicamentos não disponíveis nos serviços públicos, em razão de preços abusivos praticados pelos fabricantes ou de falta de estoque, padronização do uso, registro no país e comprovação científica de eficácia.

Na criação do Sistema Único de Saúde (SUS) foi previsto o direito do cidadão à assistência farmacêutica, mas esse direito só foi regulamentado após dez anos, com a publicação da Política Nacional de Medicamentos (PNM). Esta fortalece os princípios e as diretrizes do SUS, objetivando ainda a garantia da eficácia e segurança no uso racional de medicamentos e o acesso da população aos medicamentos essenciais 2 .

Em 2004, foi aprovada, pelo Conselho Nacional de Saúde (CNS), a Política Nacional de Assistência Farmacêutica (PNAF), cujas diretrizes se baseiam nas doutrinas do SUS e num conceito abrangente de assistência farmacêutica. A PNAF foi estabelecida como parte integrante da Política Nacional de Saúde, envolvendo um conjunto de ações voltadas à promoção e recuperação da saúde, garantindo os princípios da universalidade, integralidade e eqüidade 3 .

Assim, com base nas doutrinas e diretrizes e em suas políticas públicas relacionadas com a assistência farmacêutica, o SUS, em seus vinte anos de existência, vem se organizando para fornecer medicamentos essenciais à população. A padronização e descentralização da compra tornam mais ágeis e racionais a aquisição e dispensação dos medicamentos à população. Desta maneira, o Ministério da Saúde 4, a SES-SP 5 e a Secretaria Municipal de Saúde de São Paulo (SMS-SP) 6 criaram, com base em dados epidemiológicos, programas de dispensação de medicamentos para tratar as doenças que mais atingem a população. Os medicamentos padronizados nesses programas são avaliados no que se refere à eficiência e à efetividade, além da relação custo/benefício. Cada instância de governo tem suas competências e atribuições no gerenciamento, na operacionalização e no financiamento desses programas.

Além da questão orçamentária assinalada, muitas das decisões judiciais infringem ou distorcem princípios do SUS, sobretudo o da eqüidade.

As ações judiciais para a obtenção de medicamentos não se relacionam diretamente aos princípios da universalidade e ao princípio da integralidade uma vez que não decorrem nem de restrições e nem de exclusões estabelecidas pelo SUS. O acesso ao tratamento está garantido, seja qual for o tipo e a complexidade do problema apresentado. Entretanto, a maioria das ações ajuizadas contra a SES-SP demanda exclusivamente o acesso a produtos não previstos nos protocolos e programas executados pelo SUS. Desse modo, o acolhimento dessas demandas, no mais das vezes significa o estabelecimento de privilégios para aqueles indivíduos com poder aquisitivo para contratar um advogado e acionar o Estado.

A igualdade de condições, preconizada para a assistência, deveria impedir a existência de privilégios ou preconceitos de qualquer espécie nos serviços de saúde. No entanto, muitas ordens judiciais ferem este conceito por garantir a poucos indivíduos determinados serviços que não são oferecidos pelo SUS, beneficiando-os. Tais decisões interferem de forma negativa na organização do SUS 7 .

Este trabalho caracterizou as demandas judiciais para obtenção de medicamentos junto à SES-SP. As hipóteses que orientaram a investigação foram: (a) há interferência do poder judiciário na esfera executiva das políticas públicas de saúde; (b) essa interferência do poder judiciário na política de saúde rompe princípios básicos do SUS, especialmente a eqüidade no acesso a bens e serviços.

\section{Metodologia}

O presente estudo é um trabalho descritivo baseado na análise dos dados extraídos do Sistema de Controle Jurídico (SCJ), registro eletrônico das demandas, construído pela SES-SP para acompanhar o cumprimento das determinações judiciais.

Os dados selecionados para a análise de cada processo foram: número do processo judicial; advogado; médico prescritor; medicamento solicitado; nome do paciente; endereço de residência do paciente informado no processo; e origem da receita médica.

Como o SCJ é um registro informatizado que não foi organizado como um banco de dados para que as análises de interesse pudessem ser feitas, foi necessário padronizar cada uma das variáveis e codificá-las de forma a poder constituir um arquivo de consultas para a pesquisa a partir dos relatórios extraídos do sistema.

Definiram-se como universo da pesquisa as ações judiciais cadastradas de $1^{\circ}$ de janeiro a 31 de dezembro de 2006, movidas por pacientes que, no processo judicial, informaram residir na cidade de São Paulo e receberam medicamentos por meio de processos contra a SES-SP. 
As variáveis construídas para o estudo foram:

a) Representação jurídica privada ou estatal (Procuradoria do Estado e Ministério Público);

b) Características dos medicamentos requeridos na ação segundo disponibilidade para venda no país (possuir registro na Agência Nacional de Vigilância Sanitária - ANVISA - e preço aprovado na Câmara dos Medicamentos - CMED); classe terapêutica (segundo as normas farmacológicas da Anatomical Therapeutic Chemical Classification 8 - ATC - da Organização Mundial da Saúde - OMS) e pertinência a um dos programas de assistência farmacêutica do SUS. Todas essas informações referem-se ao ano 2006;

c) Características do paciente, incluindo origem da receita médica (rede do SUS ou sistema complementar) e local de moradia do paciente informado no processo, classificado segundo o distrito administrativo de residência (Prefeitura da Cidade de São Paulo. http://www.capital. sp.gov.br/portalpmsp/homec.jsp, acessado em 05/Mai/2008).

As análises das características dos medicamentos foram feitas inicialmente no total de itens solicitados nos processos judiciais; posteriormente foram agrupados os itens de medicamentos que geraram mais de trinta processos.

Para avaliar a extensão do processo de judicialização, avaliaram-se o número de ações, a representação jurídica e as características dos medicamentos solicitados.

$\mathrm{Na}$ análise da quebra do princípio da eqüidade utilizaram-se os dados de origem da receita médica, da área de residência referida aos estratos de vulnerabilidade social e do tipo de medicamento mais solicitado em cada estrato.

Os 96 distritos de residência foram classificados em seis estratos sociais, recorrendo-se ao Índice Paulista de Vulnerabilidade Social (IPVS). Os processos analisados envolviam 2.712 pacientes, dos quais 2.604 com endereço na cidade de São Paulo. Houve 108 perdas (entre endereços mal preenchidos ou localizados fora do Município de São Paulo).

Entende-se por vulnerabilidade social um conjunto de fatores capaz de mensurar a deterioração do nível de bem-estar, em conseqüência da exposição de pessoas, famílias ou comunidades a determinados tipos de riscos. O IPVS é um indicador desenvolvido pela Fundação Sistema Estadual de Análise de Dados (Fundação Seade), que classifica os setores censitários em seis grupos de vulnerabilidade social: nenhuma vulnerabilidade, vulnerabilidade muito baixa, vulnerabilidade baixa, vulnerabilidade média, vulnerabilidade alta e vulnerabilidade muito alta - estratos de 1 a 6 , respectivamente 9 .
O índice foi elaborado segundo uma tipologia derivada da combinação entre as categorizações sócio-econômica e demográfica ${ }^{9}$. Os distritos foram classificados nos estratos de vulnerabilidade social segundo a predominância de setores censitários de determinado estrato.

\section{Análise dos dados}

Valemo-nos de estatísticas descritivas para a caracterização das ações segundo os aspectos referidos. Os programas Epi Info, versão 3.3.2 (Centers for Disease Control and Prevention, Atlanta, Estados Unidos), e Excel 2003 (Microsoft Corp., Estados Unidos) foram utilizados para o processamento, permitindo a análise dos dados, a tabulação e a montagem dos gráficos e das tabelas da pesquisa.

Os dados analisados são apresentados em gráficos e tabelas na seção de resultados.

\section{Aspectos éticos}

O projeto foi avaliado e aprovado pelo Comitê de Ética em Pesquisa da Santa Casa de Misericórdia de São Paulo, em conformidade com as normas previstas na Resolução $n^{o}$. 196/06 do Conselho Nacional de Saúde (projeto no. 262/07). Os dados utilizados não permitirão, de nenhuma forma, a identificação dos sujeitos demandantes, nem a identificação nominal de prescritores ou advogados.

\section{Resultados}

No período estudado foram cadastrados 3.007 processos judiciais, relativos a 2.712 pacientes diferentes. Desse total, 80 (2,66\%) não tinham registro do nome do advogado no SCJ, resultando em 2.927 processos analisados neste estudo. A representação jurídica dessas ações foi privada em $74 \%$ dos casos e estatal em $26 \%$ (Procuradoria do Estado ou Ministério Público).

Foram solicitados 954 itens diferentes de medicamentos, dos quais 28 (3\%) não estavam disponíveis para venda no mercado nacional e $77 \%$ não pertenciam aos programas de assistência farmacêutica do SUS (Tabela 1). Dos itens fornecidos pelo SUS (23\% do total de itens), 120 (13\%) pertenciam ao Programa de Medicamentos de Dispensação Excepcional.

Analisados os dados referentes aos itens de medicamentos que geraram mais de trinta processos levados em consideração neste estudo, encontraram-se 42 , dos quais 4 (10\%) não esta- 
Tabela 1

Proporção dos itens de medicamentos solicitados nas ações judiciais em relação à presença ou não nos programas de assistência farmacêutica do Sistema Único de Saúde (SUS). Município de São Paulo, Brasil, 2006.

\begin{tabular}{|c|c|c|}
\hline Medicamentos solicitados & $\begin{array}{c}\text { Freqüência dos itens que } \\
\text { geraram mais de } 30 \text { processos }\end{array}$ & $\begin{array}{l}\text { Freqüência total } \\
\text { dos itens }\end{array}$ \\
\hline Itens de medicamentos que pertencem aos programas de assistência farmacêutica do SUS & $16(38,10 \%)$ & $215(22,54 \%)$ \\
\hline Itens de medicamentos não pertencentes aos programas de assistência farmacêutica do SUS & $26(61,90 \%)$ & $739(77,46 \%)$ \\
\hline Total & $42(100,00 \%)$ & $954(100,00 \%)$ \\
\hline
\end{tabular}

Fonte: Sistema de Controle Jurídico, Secretaria de Estado da Saúde de São Paulo, 2008.

vam disponíveis para venda no mercado nacional e 26 (62\%) não pertenciam aos programas de assistência farmacêutica do SUS (Tabela 1). Dos itens fornecidos pelo SUS, 5 (12\%) faziam parte do Programa de Medicamentos de Dispensação Excepcional.

Em relação à classificação terapêutica dos medicamentos solicitados segundo as normas da ATC 8, verificou-se que: $17 \%$ dos itens eram relacionados com o aparelho digestivo e o metabolismo; $17 \%$, com o sistema cardiovascular; e $16 \%$, com o sistema nervoso (Tabela 2 ).

Considerando-se a classe terapêutica dos medicamentos que geraram mais de trinta processos, detectou-se que: a freqüência foi de $33 \%$ para antineoplásicos e agentes imunomoduladores; $31 \%$, para o aparelho digestivo e o metabolismo; e 19\%, para o sistema cardiovascular (Tabela 2).

Com relação ao local de prescrição, $48 \%$ dos pacientes possuíam receitas médicas de estabelecimentos da rede do SUS; $47 \%$, do sistema complementar; e em $4 \%$ dos atendimentos não foi possível classificar a origem da receita.

Segundo a classificação do local de moradia do paciente informado no processo pelo IPVS, $16 \%$ dos solicitantes pertenciam ao estrato 1 ; $35 \%$, ao estrato 2; e $22 \%$, ao estrato 3 (Figura 1).

Os medicamentos que geraram mais de 30 processos estudados foram agrupados em oito grupos, conforme a Tabela 3. Foi feita a distribuição desses grupos de medicamentos segundo o local de moradia dos pacientes que ajuizaram a ação, classificando-se por estrato de IPVS (Figura 2). Observa-se que os residentes nas regiões com IPVS 2 representam a maior concentração em praticamente todos os itens de medicamentos, com exceção dos residentes nas áreas com IPVS 3, que têm maior concentração de pedidos entre as insulinas padronizadas pelo Ministério da Saúde pertencentes ao grupo 2 de medicamentos.

\section{Discussão}

A eqüidade em saúde refere-se à diminuição das diferenças consideradas desnecessárias e evitáveis 10 . Para o SUS, todo cidadão é igual perante a lei e deve ser atendido de acordo com suas necessidades. Assim, os serviços de saúde precisam conhecer as necessidades dos diferentes grupos da população e trabalhar para satisfazê-las, oferecendo mais a quem mais precisa, contribuindo para a diminuição das desigualdades existentes.

O direito à saúde é um direto social, de acordo com a Constituição Federal, a concretização de tais direitos depende da elaboração e implementação das políticas públicas de saúde. Elas objetivam melhorar as condições de vida da população e realizar a equalização de situações desiguais. As políticas de saúde devem ser implantadas em escala coletiva, a fim de atender a esses preceitos legais 11 .

De maneira geral, os juízes, ao deferirem as ordens para o fornecimento de medicamentos como forma de garantir os direitos dos indivíduos, não observam a política de assistência farmacêutica do SUS 11,12. Essas ações têm conseqüências orçamentárias importantes, uma vez que os recursos são finitos e sua administração deve ser planejada e balizada pelas políticas de saúde. Conseqüentemente, as demandas judiciais prejudicam essas políticas, impedindo a alocação racional dos escassos recursos públi$\cos 13$, além de interferir no planejamento das ações de saúde 7 .

Ao se fornecerem medicamentos por ordem judicial, não está sendo avaliado se aquele tratamento realmente é o melhor em termos de relação custo/benefício, se o indivíduo realmente necessita do medicamento pleiteado e este não pode ser substituído por outro disponível nos programas de assistência farmacêutica do SUS, se o paciente tem condições financeiras de pagar o tratamento ou, até mesmo, o advogado e, 
Classificação dos medicamentos segundo a classe terapêutica e freqüência das solicitações.

\begin{tabular}{|c|c|c|}
\hline Classe terapêutica (ATC) & $\begin{array}{c}\text { Freqüência dos itens que } \\
\text { geraram mais de } 30 \text { processos }\end{array}$ & $\begin{array}{l}\text { Freqüência total } \\
\text { dos itens }\end{array}$ \\
\hline Aparelho digestivo e metabolismo & $13(30,95 \%)$ & $166(17,40 \%)$ \\
\hline Sistema cardiovascular & $8(19,05 \%)$ & $166(17,40 \%)$ \\
\hline Sistema nervoso & $1(2,38 \%)$ & $152(15,93 \%)$ \\
\hline Sistema respiratório & $1(2,38 \%)$ & $80(8,39 \%)$ \\
\hline Antineoplásico e agente imunomoduladores & $14(33,34 \%)$ & $73(7,65 \%)$ \\
\hline Sistema músculo-esquelético & $1(2,38 \%)$ & $44(4,61 \%)$ \\
\hline Sangue e órgãos hematopoéticos & $1(2,38 \%)$ & $43(4,51 \%)$ \\
\hline Agente anti-infeccioso para uso sistêmico & $1(2,38 \%)$ & $40(4,19 \%)$ \\
\hline Dermatológico & - & $39(4,09 \%)$ \\
\hline Órgãos dos sentidos & $1(2,38 \%)$ & $39(4,09 \%)$ \\
\hline Hormônios de uso sistêmico, exceto hormônios sexuais & $1(2,38 \%)$ & $33(3,46 \%)$ \\
\hline Sistema geniturinário e hormônios sexuais & - & $21(2,20 \%)$ \\
\hline Vários & - & $8(0,84 \%)$ \\
\hline Produtos antiparasitários & - & $4(0,42 \%)$ \\
\hline Medicamentos manipulados * & - & $46(4,82 \%)$ \\
\hline Total & 42 (100,00\%) & 954 (100,00\%) \\
\hline
\end{tabular}

ATC: Anatomical Therapeutic Chemical Classification.

* Os medicamentos manipulados não foram classificados pela ATC.

Fonte: Sistema de Controle Jurídico, Secretaria de Estado da Saúde de São Paulo, 2008.

ainda, se não estão sendo infringidos alguma lei ou algum princípio fundamental do sistema de saúde. Apenas se cumpre a ordem determinada pelo juiz.

A maioria das ações analisadas foi ajuizada por advogados particulares. A justiça gratuita contribuiu tão-somente com $1 / 4$ das ações. $\mathrm{Na}$ maioria dos trabalhos publicados no Brasil predominam ações conduzidas pela representação jurídica estatal 14,15,16, ao contrário do descrito neste trabalho. $\mathrm{O}$ fato de essas ações geralmente serem ajuizadas por advogados particulares mostra que os pacientes arcaram com os custos dessa representação e em princípio poderiam adquirir os medicamentos solicitados.

A Defensoria do Estado, criada pela Lei Complementar $n^{\circ} .988$, de 9 de janeiro de 2006, tem a finalidade de prestar assistência judicial às pessoas carentes do Estado de São Paulo. Anteriormente, a Procuradoria do Estado possuía uma equipe de advogados somente para atender cidadãos sem recursos financeiros que necessitavam do Poder Judiciário. No período de coleta de dados deste trabalho ainda eram os procuradores do Estado que impetravam as ações contra o Estado em nome da população sem recursos.

Os direitos sociais, como já abordado, devem ser realizados coletivamente, a fim de que haja acesso igualitário e universal dos indivíduos aos benefícios oferecidos pelas políticas públicas. Terrazas 1, ao analisar decisões de solicitações de medicamento para tratamento de hepatite $\mathrm{C}$ junto à Seção de Direito Público do Tribunal de Justiça do Estado de São Paulo, na Comarca da Capital, detectou que, das decisões proferidas entre 1998 a 2005, 98\% se referiam a processos individuais e somente $2 \%$ a ações coletivas. A maioria dos pacientes dessas ações utilizou a justiça particular para solicitar tais medicamentos. Assim, foram beneficiadas pela intervenção do Poder Judiciário pessoas que possuem melhores condições sócio-econômicas e com acesso à informação. Com essas demandas judiciais, pretende-se garantir direitos sociais que são essencialmente coletivos. Entretanto, como a atuação do Poder Judiciário ocorre no âmbito individual, isto é, de forma individualizada, conforme demonstrado por Terrazas e também pelos resultados do presente trabalho, acaba havendo tratamento diferenciado, privilegiando esses pacientes, o que é incompatível com a idéia de igualdade proposta pelo SUS.

Em relação aos medicamentos solicitados, verificou-se que cerca de $77 \%$ não pertenciam aos programas de assistência farmacêutica do SUS. Dos demais itens, aproximadamente $12 \%$ 
Distribuição proporcional dos pacientes com ação judicial solicitando medicamentos, por estrato do Índice Paulista de Vulnerabilidade Social (IPVS) de residência (2006), comparando com a população total (2004). Município de São Paulo, Brasil, 2006.

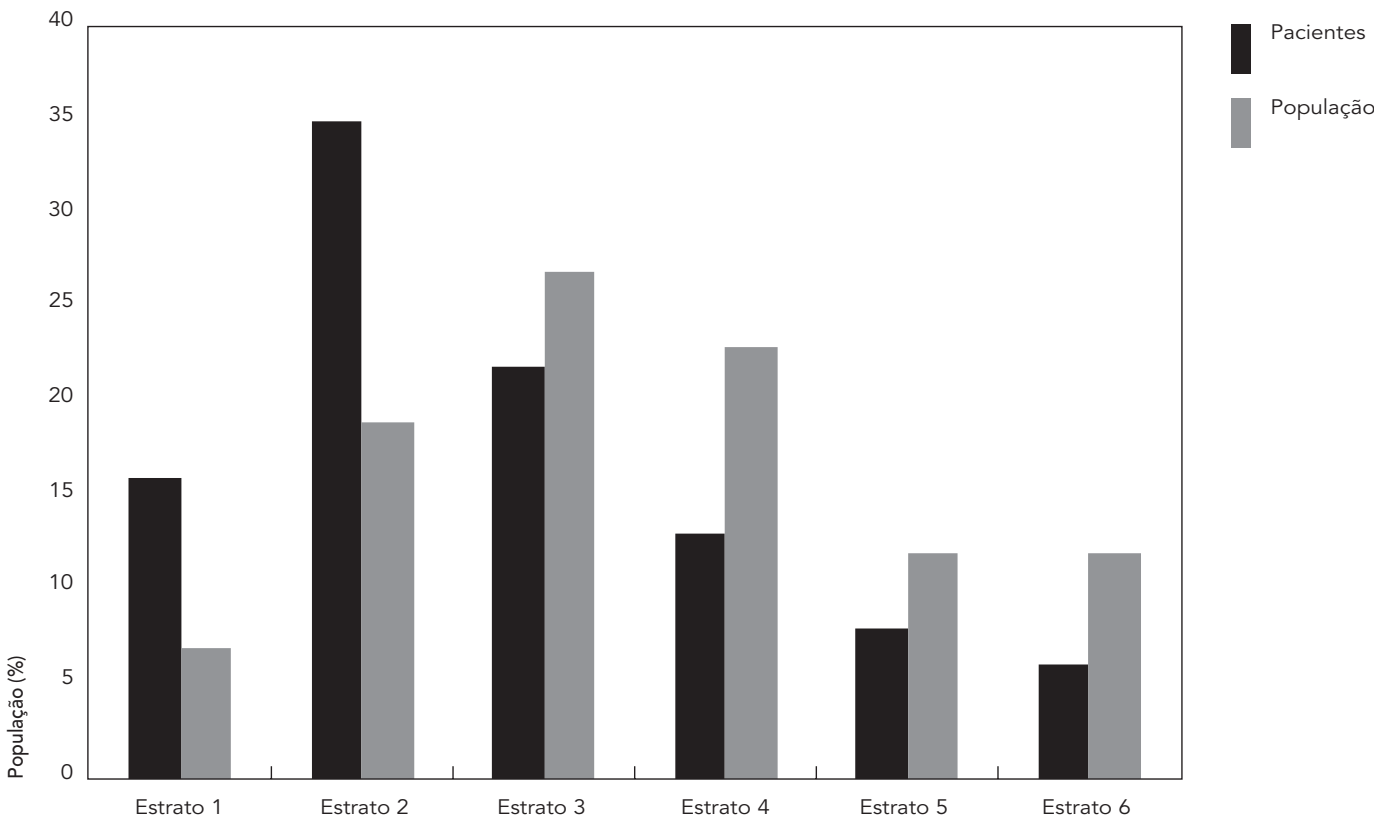

Nota: estrato 1: nenhuma vulnerabilidade; estrato 2: vulnerabilidade muito baixa; estrato 3: vulnerabilidade baixa; estrato 4 vulnerabilidade média; estrato 5: vulnerabilidade alta; estrato 6: vulnerabilidade muito alta.

Fonte: Sistema de Controle Jurídico, Secretaria de Estado da Saúde de São Paulo, 2008

eram do Programa de Medicamentos de Dispensação Excepcional.

Vieira \& Zucchi 15, analisando as ações referentes ao acesso a medicamentos do Município de São Paulo no ano 2005, mostraram que $62 \%$ dos itens solicitados faziam parte de listas de medicamentos do SUS, e, dentre eles, $15 \%$ pertenciam ao Programa de Medicamentos Excepcionais. No Estado do Rio de Janeiro, segundo Messeder et al. 14, 30\% dos medicamentos foram solicitados por ação judicial sem financiamento definido pelo SUS, vale dizer, não pertenciam aos programas de medicamentos. Dos itens com financiamento, cerca de $1 / 3$ fazia parte do Programa de Medicamentos de Dispensação Excepcional. Já em Brasília, no ano 2006, de acordo com Santos \& Gonçalves 16, metade dos itens solicitados era padronizado, e 1/4 se referia a Medicamentos Excepcionais. Portanto, a situação do Estado de São Paulo é diferente da dos demais locais estudados.

O fato de $38 \%$ dos medicamentos que geraram mais de trinta processos analisados por este trabalho pertencerem a algum programa de fornecimento do SUS pode indicar a falta de conhecimento dos médicos quanto aos programas e/ou irregularidade no fornecimento desses itens. Pelo menos $47 \%$ das prescrições foram provenientes de estabelecimentos particulares, o que também pode indicar que seus médicos não estão familiarizados com as regras do SUS.

Os dados relativos ao Estado de São Paulo, sistematizados por este estudo, sugerem que a população tem acesso aos medicamentos referentes aos programas e não tem ocorrido descontinuidade de abastecimento, pois a maioria das solicitações se liga a medicamentos não incluídos nos programas. É importante lembrar ainda que os pedidos judiciais podem se referir a receitas médicas que são compostas de vários itens, podendo essas receitas conter medicamentos disponíveis pelos programas de assistência farmacêutica do SUS, explicando-se, assim, a sua presença nas demandas judiciais. Por exemplo, é comum uma prescrição de insulina especial estar associada à insulina humana 
Classificação dos medicamentos que geraram mais de trinta processos, por princípio ativo em grupos.

\begin{tabular}{|c|c|c|}
\hline Grupo & Características & Medicamentos \\
\hline \multirow[t]{2}{*}{1} & Medicamentos básicos fornecidos & Ácido Acetilsalicílico 100mg, Captopril, Furosemida, \\
\hline & nas unidades básicas de saúde & Glibenclamida, Hidroclorotiazida \\
\hline 2 & Insulinas fornecidas nas unidades básicas de saúde & Insulina Humana NPH, Insulina Humana Regular \\
\hline \multirow[t]{2}{*}{3} & Medicamentos não disponíveis para venda no & Bevacizumabe, Cetuximabe, Erlotinibe \\
\hline & mercado nacional em 2006 (oncológicos) & \\
\hline 4 & Insulinas especiais & Insulina Aspart, Insulina Glargina, Insulina Lispro \\
\hline \multirow[t]{2}{*}{5} & Medicamentos pertencentes ao Programa de & Infliximabe, Alfapeginterferona 180mcg, Ribavirina, \\
\hline & Medicamentos de Dispensação Excepcional & Sinvastatina10 mg, Levotiroxina Sódica 100mcg \\
\hline 6 & Medicamentos oncológicos & Anastrozol, Imatinibe 100mg, Rituximabe, Temozolomida \\
\hline 7 & Medicamentos imunobiológicos & Adalimumabe, Etanercepte \\
\hline 8 & Outros medicamentos & $\begin{array}{l}\text { Aripiprazol 15mg, Enalapril, Glucagon, Losartana Potássica 50mg, Metformina, } \\
\text { Omeprazol 20mg, Palivizumabe, Teriparitida, Timolol, Brometo de Tiotrópio }\end{array}$ \\
\hline
\end{tabular}

Figura 2

Distribuição dos processos por estrato do Índice Paulista de Vulnerabilidade Social (IPVS) de residência dos solicitantes e tipo de medicamento solicitado.

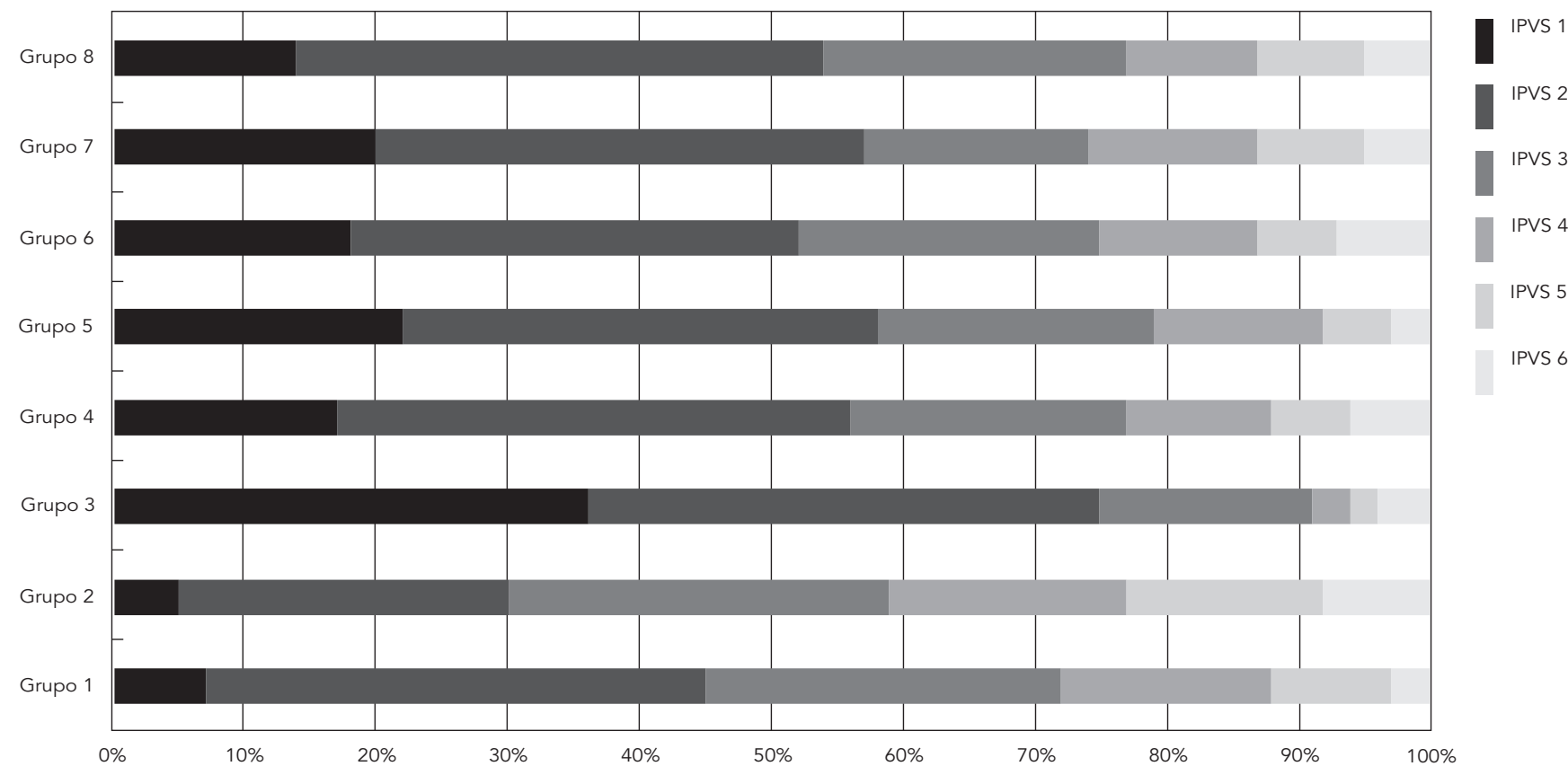

Nota: ver Tabela 3 para descrição dos grupos.

Fonte: Sistema de Controle Jurídico, Secretaria de Estado da Saúde de São Paulo, 2008. 
NHP ou regular, ao ácido acetilsalicílico de 100mg e a uma atorvastatina ou sinvastatina. Apesar de neste caso somente as insulinas especiais não estarem disponíveis no SUS, o paciente solicita todos os medicamentos que compõem a receita original, mesmo achando-se os demais acessíveis pelas vias regulares de distribuição de medicamentos.

Em relação aos medicamentos excepcionais, não foi verificado neste estudo se as prescrições estavam de acordo com os protocolos e as diretrizes terapêuticas do Ministério da Saúde. Freqüentemente, nas ações judiciais, medicamentos padronizados para uso em determinadas doenças são prescritos para outras situações não previstas pelos protocolos. A título de exemplo, observase a prescrição de infliximabe para psoríase, e no protocolo a indicação desse medicamento é para doença de Crohn (CID-10 K50, K50.0, K50.1, K50.8 e K50.9) e obedece a critérios de inclusão e exclusão de tratamento 17. Desta forma, pode haver solicitação que não seja preconizada pelo Ministério da Saúde, gerando-se, desse modo, a demanda judicial.

Em relação à classificação segundo a classe terapêutica dos medicamentos, observou-se no presente trabalho uma freqüência de $17 \%$ para medicamentos com atuação no aparelho digestivo e no metabolismo, além do sistema cardiovascular, e $16 \%$ para o sistema nervoso. Messeder et al. 14 relataram que $21 \%$ dos medicamentos foram utilizados para o sistema nervoso central; $17 \%$, para doenças cardiovasculares; $16 \%$, para o trato alimentar ou o metabolismo, além de três grupos de medicamentos de uso contínuo, para tratamentodedoenças crônicas.Vieira\&Zucchi15, por sua vez, descreveram como doenças mais freqüentemente referidas o diabetes e o câncer, com proporções, respectivamente, de $46 \%$ e $22 \%$, também enfermidades crônicas que têm custo de tratamento elevado, pois o paciente recebe o tratamento por um período longo.

Nos nossos achados a classificação segundo a classe terapêutica dos medicamentos, para os itens que geraram mais de trinta processos, mostrou que cerca de $1 / 3$ deles se refere a antineoplásicos e agentes imunomoduladores; $1 / 3$ tem ação sobre o aparelho digestivo e o metabolismo; e 1/5 se destina ao sistema cardiovascular.

O envelhecimento da população é um fenômeno mundial. No Brasil, o número de idosos está aumentando de forma rápida, com menores de 15 anos perdendo representatividade e maiores de 65 anos aparecendo em quantidade crescente 18 . A terceira idade apresenta um quadro de enfermidades complexas e onerosas, caracterizado por doenças crônicas e múltiplas, com exigência de medicação de uso contínuo 19.
O envelhecimento da população em geral poderia explicar o fato de a maior parte dos medicamentos solicitados serem de uso contínuo e uma grande parte se destinar ao tratamento do câncer, mal que normalmente é mais freqüente em indivíduos idosos. No presente trabalho, considerando a origem das receitas médicas, aproximadamente metade dos pacientes possuía receitas médicas provenientes de estabelecimentos do sistema complementar. Vieira \& Zucchi 15 encontraram cerca de $70 \%$ das receitas prescritas por entidades da rede SUS; na pesquisa de Messeder et al. 14, a proporção foi de $80 \%$; e na de Santos \& Gonçalves $16,60 \%$.

Os pedidos encaminhados pela Procuradoria do Estado podem de alguma forma enviesar este dado, já que se exigia do assistido que ele possuísse uma receita médica prescrita em órgão público. Logo, mesmo que o medicamento tivesse sido prescrito por médico de serviço particular, para propor ação judicial por intermédio da Procuradoria, o assistido devia recorrer ao SUS e solicitar um relatório médico e uma receita prescrevendo o medicamento desejado, ocorrendo, desta maneira, a transcrição da receita. Este achado está em concordância com observações realizadas em outros estudos 15. Dos 2.927 processos analisados, 762 (1/4) foram impetrados pela Defensoria Pública. Não se pode afirmar o número real de transcrição de receitas feitas em estabelecimentos do SUS. Provavelmente esse número está superestimado para essa fração dos processos.

Quanto à classificação, segundo o IPVS, do endereço informado pelos pacientes no processo judicial, constatou-se que a maioria reside no estrato 2, caracterizando-se uma sobre-representação desse estrato. Comparando a freqüência acumulada, verificou-se que aproximadamente $74 \%$ dos pacientes residem em áreas pertencentes aos estratos 1, 2 e 3; já na população em geral esta porcentagem é de $53 \%$. Considerando apenas os estratos 1 e 2, os mais bem aquinhoados da população, a diferença entre a parcela atendida pelas ações judiciais é o dobro de sua distribuição na população geral. Esses dados demonstram que a população com maior poder aquisitivo é que está se beneficiando do resultado das ações judiciais.

Em razão disso, as demandas judiciais estão ferindo o princípio da eqüidade do SUS, ou seja, as ações judiciais não estão fornecendo medicamentos a quem utiliza exclusiva ou preferencialmente o sistema público de saúde e depende do fornecimento gratuito de medicamentos, exatamente as pessoas residentes nos estratos de vulnerabilidade mais alta. Nesses estratos de maior vulnerabilidade, os setores apresentam nível mé- 
dio e baixo na dimensão sócio-econômica, com baixa escolaridade e menores médias de rendimentos do responsável pelo domicílio ${ }^{9}$.

Segundo os dados do presente trabalho, medicamentos dos grupos 3 e 6 são solicitados em maior freqüência pelos pacientes residentes nos distritos administrativos pertencentes aos estratos 1 e 2 do IPVS (75\% nos oncológicos não disponíveis para venda no país e $52 \%$ nos oncológicos disponíveis). Nesses estratos, a média de idade (em anos), o rendimento nominal médio (em Reais) e a escolaridade do responsável pelo domicílio são maiores que para os demais estratos, segundo dados da Fundação Seade 9 . A situação sugere que o envelhecimento da população, melhores condições sócio-econômicas e o acesso à informação aumentem os pedidos de medicamentos oncológicos. Este fato tornase mais evidente quando os medicamentos não estão disponíveis para venda no país. No SUS, o paciente com diagnóstico de câncer tem atendimento integral em unidades ou centros especializados e habilitados pelo Ministério da Saúde para o tratamento. As demandas judiciais transformam o sistema de saúde em um mero fornecedor de medicamentos. No SUS, a assistência farmacêutica tem de estar vinculada à assistência médica, com as demandas judiciais isso não ocorre, havendo apenas o fornecimento do medicamento, independentemente da origem da assistência médica.

As insulinas do grupo 2, fornecidas pelo Ministério da Saúde (tratamento padrão para o paciente insulinodependente), concentram os pedidos entre os pacientes residentes nos estratos 3 (29\%). Porém, as insulinas especiais (grupo 4) estão concentradas no estrato 2 (39\%), demonstrando novamente que o nível sócio-econômico mais elevado e o maior acesso à informação estão relacionados com as prescrições mais sofisticadas dos medicamentos.

Vieira \& Zucchi 15 classificaram, no Município de São Paulo, o local de moradia dos pacientes pelo índice de exclusão/inclusão, detectando que $62 \%$ dos indivíduos residem em áreas com menor grau de exclusão social. Seu estudo conclui que "são os indivíduos menos carentes de proteção social que estão movendo ações contra o poder público municipal e instala a dúvida sobre o descumprimento das ações de eqüidade propostas pelo SUS" 15 (p. 221).

A análise da distribuição dos processos por estrato do IPVS de residência dos solicitantes e por tipo de medicamento solicitado, bem como a classificação de residência dos pacientes pelo IPVS reforçam a hipótese de que a interferência do Poder Judiciário na política de saúde rompe o princípio da eqüidade ao favorecer as deman- das dos que menos necessitam, em detrimento daqueles que só podem contar com o sistema público de saúde, ampliando a ineqüidade já existente.

As ações judiciais podem ser um canal legítimo de defesa dos direitos fundamentais dos indivíduos à medida que o Estado não implementa de maneira adequada as políticas públicas. Todavia, ao verificar que a maioria dos medicamentos solicitados nessas demandas não pertence aos programas de medicamentos preconizados pelo SUS, isto é, que não são padronizados pelos protocolos do sistema público de saúde, podese dizer que as ações judiciais ignoram o que a política de assistência farmacêutica normalizou. Esse fato pode sugerir também a falta ou demora de incorporação de novos medicamentos nas listas de medicamentos padronizados. No caso do Programa de Medicamentos de Dispensação Excepcional, por exemplo, houve uma incorporação expressiva de novos itens em 200220 e depois em 200621 e 200822 .

O processo judicial é um espaço limitado e inadequado para considerar decisões a respeito dos direitos sociais, pois elas são complexas e abordam questões coletivas e não individuais como a maioria das demandas judiciais. Além do mais, o processo força o deslocamento de recursos públicos das políticas de saúde para o atendimento das demandas individualizadas. Assim, busca-se uma solução individualizada de uma questão coletiva. Segundo Octávio Luiz Motta Ferraz (2005, apud Terrazas 1, p. 16-7), “o litígio tende a se focar no caso individual e desconsidera o quadro geral, sendo indiferente aos interesses que necessariamente serão afetados pela decisão judicial. Isso resulta no problema da não-representatividade, que traz questões não somente sobre competência institucional, mas também sobre justiça".

Outra questão que também precisa ser pontuada é a falta de conhecimento técnico dos juízes para tomarem decisões referentes aos direitos sociais 1 . Apesar de o Judiciário solicitar informações relevantes em relação às demandas judiciais antes de dar um veredicto, caso elas não estejam no processo, o problema é de que forma elas chegam e são interpretadas.

\section{Limitações do estudo}

Este trabalho analisou somente as ordens judiciais ajuizadas na SES-SP e atendidas na cidade de São Paulo. As demais cidades do estado não estão contempladas, pois, quando da realização do estudo, o SCJ possuía somente os dados completos da capital. 
O SCJ foi implantado em 2005 e desde então passou por atualizações com o objetivo de melhorar seu desempenho. No primeiro momento os processos foram cadastrados com o histórico dos pacientes existente no local onde era efetuada a dispensação dos medicamentos/produtos envolvidos em ação judicial. Inicialmente, muitos dados precisavam ser digitados no sistema, com ocorrência de erros de digitação.

Outra limitação do estudo foi denotar diferença entre a determinação judicial e o que estava sendo efetivamente atendido pela SES-SP. As novas ações passam a ser incluídas no SCJ assim que ocorre a notificação à SES-SP. Para diminuir o erro de digitação, as atualizações do SCJ têm menos campos a serem preenchidos e mais campos padronizados.

Um último óbice encontrado foi a impossibilidade de acesso a determinadas informações pelo fato de elas estarem ilegíveis ou mesmo indisponíveis no processo.

No período de desenvolvimento deste estudo, aumentou o cadastramento, no SCJ, de pacientes com solicitação de medicamentos para diabetes, os quais já estavam sendo atendidos, contudo ainda não se achavam cadastrados no sistema. Em 2006, os medicamentos adalimumabe e etanercepte foram incluídos no protocolo do Programa de Medicamentos de Dispensação Excepcional 16, mas, como isso ocorreu no final do ano, eles foram por nós tratados como não padronizados.

Em relação aos endereços dos pacientes, foram utilizados os que estavam cadastrados no
SCJ, ou seja, os que constavam no processo judicial. No entanto, não se pode saber ao certo se se trata realmente dos endereços de residência do paciente.

\section{Conclusão}

A análise dos processos referentes à dispensação de medicamentos mediante demandas judiciais permitiu evidenciar os efeitos que essas decisões têm sobre a formulação e a execução da política de saúde. Os dados mostram que a parcela da população atendida por demandas judiciais tem em média melhores condições sócio-econômicas, residindo em áreas com baixa ou sem nenhuma vulnerabilidade social.

A despeito das intenções de arbitragem de problemas sociais e de defesa dos interesses dos indivíduos diante do poder do Estado, a interferência do Judiciário acaba por atender aos indivíduos que por sua inclusão social já se encontram em posição privilegiada, reforçando ainda mais as desigualdades sociais no campo da saúde.

A interpretação dos direitos sociais apenas na dimensão individual, desconsiderando a dimensão coletiva, não permite considerar o problema em todas as suas dimensões e em toda a sua complexidade, o que resulta em medidas que em vez de promoverem a justiça social acabam prolongando indefinidamente a imensa dívida social com a parcela mais vulnerável da população.

\section{Resumo}

O fornecimento de medicamentos por ação judicial, pela Secretaria de Estado da Saúde de São Paulo (SES$S P)$, tornou-se uma prática rotineira e cada vez mais freqüente. A judicialização do sistema de saúde entra em confronto com princípios básicos do SUS, como o da eqüidade. Este trabalho caracterizou as demandas judiciais para obtenção de medicamentos sob a ótica da judicialização da política pública e da quebra do princípio da eqüidade na dimensão coletiva. O estudo é um trabalho descritivo dos processos judiciais de solicitação de medicamentos à SES-SP relacionados no Sistema de Controle Jurídico (SCJ) no ano 2006. A maioria das ações analisadas foi ajuizada por advogados particulares; $47 \%$ dos pacientes possuíam receitas da rede privada e cerca de $73 \%$ dos processos foram provenientes de pacientes residentes nos três estratos de menor vulnerabilidade social do Município de São Paulo, Brasil. Os dados demonstram que essas demandas judiciais não respeitam princípios do SUS como o da eqüidade, privilegiando indivíduos com melhor poder aquisitivo e com acesso à informação.

Decisões Judiciais; Assistência Farmacêutica; Medicamentos; Direito à Saúde 


\section{Colaboradores}

A. L. Chieffi realizou a busca bibliográfica, a coleta e a análise de dados, a discussão dos resultados e a redação do artigo. R. B. Barata contribuiu com a revisão da análise de dados, da análise dos resultados, da discussão e do texto final.

\section{Referências}

1. Terrazas FV. O poder judiciário como voz institucional dos pobres: o caso das demandas judiciais por medicamentos [Dissertação de Mestrado]. São Paulo: Faculdade de Direito, Universidade de São Paulo; 2008.

2. Ministério da Saúde. Portaria no ${ }^{\circ}$ 3.916, de 30 de outubro de 1998. Dispõe sobre a aprovação da Política Nacional de Medicamentos. Diário Oficial da União 1998; 1 out.

3. Ministério da Saúde/Conselho Nacional de Saúde. Resolução no ${ }^{\circ}$. 338, de 6 de maio de 2004. Diário Oficial da União 2004; 7 mai.

4. Ministério da Saúde. Assistência farmacêutica. http://portal.saude.gov.br/SAUDE/area.cfm?id_ area=1000 (acessado em 27/Out/2008).

5. Secretaria de Estado da Saúde de São Paulo. Assistência farmacêutica. http://portal.saude.sp.gov. $\mathrm{br} /$ content/assistencia_farmaceutica.mmp (acessado em 27/Out/2008)

6. Secretaria Municipal de Saúde de São Paulo. Assistência farmacêutica. http://www2.prefeitura. sp.gov.br/secretarias/saude/ass_farmaceutica (acessado em 27/Out/2008).

7. Santos L. SUS: contornos jurídicos da integralidade da atenção à saúde. Radis Comunicação em Saúde 2006; (49). http://www.ensp.fiocruz.br/ radis/49/web-02.html (acessado em 25/Jul/2008).

8. WHO Collaborating Centre for Drug Statistics Methodology. Anatomical Therapeutic Chemical classification. http://www.whocc.no/atcddd/ (acessado em 05/Mai/2008).

9. Fundação Sistema Estadual de Análise de Dados. IPVS - Índice Paulista de Vulnerabilidade Social. http://www.seade.gov.br/produtos/ipvs/subprefeituras.php (acessado em 06/Jun/2008).

10. Viana ALD, Fausto MCR, Lima LD. Política de saúde e equidade. São Paulo Perspect 2003; 17:58-68.

11. Marques SB, Dallari SG. Garantia do direito social à assistência farmacêutica no Estado de São Paulo. Rev Saúde Pública 2007; 41:1001-7.

12. Vieira FS. Ações judiciais e direito à saúde: reflexão sobre a observância aos princípios do SUS. Rev Saúde Pública 2008; 42:365-9.
13. Barroso LR. Da falta de efetividade à judicialização à saúde: fornecimento gratuito de medicamentos e parâmetros para a atuação judicial. Migalhas de Peso 2008. http://www.migalhas.com.br/mostra noticia_articuladas.aspx? cod $=52582$ (acessado em 08/Fev/2008).

14. Messeder AM, Osorio-de-Castro CGS, Luiza VL. Mandados judiciais como ferramenta para garantia do acesso a medicamentos no setor público: a experiência do Rio de Janeiro, Brasil. Cad Saúde Pública 2005; 21:525-34.

15. Vieira FS, Zucchi P. As distorções causadas palas ações judiciais à política de medicamentos no Brasil. Rev Saúde Pública 2007; 41:214-22.

16. Santos CC, Gonçalves AS. Análise descritiva de mandados judiciais impetrados contra a Secretaria de Saúde do Distrito Federal para fornecimento de medicamentos [Monografia]. Brasília: Curso de Especialização em Gestão de Instituições de Saúde, Fundação de Ensino e Pesquisa em Ciências da Saúde; 2006.

17. Departamento de Sistemas e Redes, Secretaria de Assistência à Saúde, Ministério da Saúde. Protocolos clínicos e diretrizes terapêuticas: medicamentos excepcionais. Brasília: Ministério da Saúde; 2002.

18. Carvalho JAM, Rodríguez-Wong LL. A transição da estrutura etária da população brasileira na primeira metade do século XXI. Cad Saúde Pública 2008; 24:597-605.

19. Veras RP. Fórum. Envelhecimento populacional e as informações de saúde do PNAD: demandas e desafios contemporâneos. Introdução. Cad Saúde Pública 2007; 23:2463-6.

20. Ministério da Saúde. Portaria no ${ }^{\circ}$ 1.318, de 23 de julho de 2002. Diário Oficial da União 2002; 24 jul.

21. Ministério da Saúde. Portaria ${ }^{\circ}$. 2.577, de 27 de outubro de 2006. Diário Oficial da União 2006; 13 nov.

22. Ministério da Saúde. Portaria no ${ }^{\circ}$ 1.869, de 04 de setembro de 2008. Diário Oficial da União 2008; 4 set.

Recebido em 21/Jan/2009

Versão final reapresentada em 23/Mai/2009 Aprovado em 15/Jun/2009 